\title{
QUEEN'S
UNIVERSITY
BELFAST
}

\section{A comb-sampling method for enhanced mass analysis in linear electrostatic ion traps}

Greenwood, J. B., Kelly, O., Calvert, C. R., Duffy, M. J., King, R. B., Belshaw, L., Graham, L., Alexander, J. D., Williams, I. D., Bryan, W. A., Turcu, I. C. E., Cacho, C. M., \& Springate, E. (2011). A comb-sampling method for enhanced mass analysis in linear electrostatic ion traps. Review of Scientific Instruments, 82(4), [043103]. https://doi.org/10.1063/1.3572331

Published in:

Review of Scientific Instruments

Document Version:

Publisher's PDF, also known as Version of record

Queen's University Belfast - Research Portal:

Link to publication record in Queen's University Belfast Research Portal

\section{Publisher rights}

Copyright 2011 American Institute of Physics. This article may be downloaded for personal use only. Any other use requires prior permission of the author and the American Institute of Physics.

The following article appeared in Rev. Sci. Instrum. 82, 043103 (2011) and may be found at http://scitation.aip.org/content/aip/journal/rsi/82/4/10.1063/1.3572331.

\section{General rights}

Copyright for the publications made accessible via the Queen's University Belfast Research Portal is retained by the author(s) and / or other copyright owners and it is a condition of accessing these publications that users recognise and abide by the legal requirements associated with these rights.

\section{Take down policy}

The Research Portal is Queen's institutional repository that provides access to Queen's research output. Every effort has been made to ensure that content in the Research Portal does not infringe any person's rights, or applicable UK laws. If you discover content in the Research Portal that you believe breaches copyright or violates any law, please contact openaccess@qub.ac.uk. 


\title{
A comb-sampling method for enhanced mass analysis in linear electrostatic ion traps
}

\author{
J. B. Greenwood, ${ }^{1}$,a) O. Kelly, ${ }^{1}$ C. R. Calvert, ${ }^{1}$ M. J. Duffy, ${ }^{1}$ R. B. King,${ }^{1}$ L. Belshaw, ${ }^{1}$ \\ L. Graham, ${ }^{1}$ J. D. Alexander, ${ }^{1}$ I. D. Williams, ${ }^{1}$ W. A. Bryan, ${ }^{2}$ I. C. E. Turcu, ${ }^{3}$ C. M. Cacho, ${ }^{3}$ \\ and E. Springate ${ }^{3}$ \\ ${ }^{1}$ Centre for Plasma Physics, School of Mathematics and Physics, Queen's University Belfast, \\ Belfast BT7 1NN, United Kingdom \\ ${ }^{2}$ Department of Physics, Swansea University, Swansea SA2 8PP, United Kingdom \\ ${ }^{3}$ Central Laser Facility, STFC Rutherford Appleton Laboratory, Didcot, Oxfordshire OX11 0QX, \\ United Kingdom
}

(Received 16 December 2010; accepted 9 March 2011; published online 8 April 2011)

\begin{abstract}
In this paper an algorithm for extracting spectral information from signals containing a series of narrow periodic impulses is presented. Such signals can typically be acquired by pickup detectors from the image-charge of ion bunches oscillating in a linear electrostatic ion trap, where frequency analysis provides a scheme for high-resolution mass spectrometry. To provide an improved technique for such frequency analysis, we introduce the CHIMERA algorithm (Comb-sampling for High-resolution IMpulse-train frequency ExtRAaction). This algorithm utilizes a comb function to generate frequency coefficients, rather than using sinusoids via a Fourier transform, since the comb provides a superior match to the data. This new technique is developed theoretically, applied to synthetic data, and then used to perform high resolution mass spectrometry on real data from an ion trap. If the ions are generated at a localized point in time and space, and the data is simultaneously acquired with multiple pickup rings, the method is shown to be a significant improvement on Fourier analysis. The mass spectra generated typically have an order of magnitude higher resolution compared with that obtained from fundamental Fourier frequencies, and are absent of large contributions from harmonic frequency components. (C) 2011 American Institute of Physics. [doi:10.1063/1.3572331]
\end{abstract}

\section{INTRODUCTION}

Electrostatic devices are increasingly being used to store ions for scientific studies and for mass spectrometry applications. ${ }^{1}$ Unlike ion traps which use magnetic or timevarying electric fields, the storage conditions in an electrostatic trap are independent of mass for ions of the same energy per charge; this allows molecules as large as proteins to be studied. While the electrostatic Kingdon trap ${ }^{2}$ has been in use for nearly 90 years, it is only in the last 20 years that a new range of electrostatic devices have been developed.

The electrostatic ion storage ring in Aarhus (ELISA) ${ }^{3}$ was one of the first of these instruments to be developed; it is much smaller than the magnetic equivalent, and has inspired the further development of cryogenic ${ }^{4,5}$ and miniature ${ }^{6}$ storage rings. Around the same time, the Kingdon trap was modified to create the Orbitrap, ${ }^{7}$ for which the mass resolution reduces more slowly as a function of mass than with other high performance mass spectrometers, such as ion cyclotron resonance (ICR) devices.

The linear electrostatic ion trap (LEIT), which acts as an analogue to a laser cavity, is another innovation in electrostatic storage, ${ }^{8-10}$ with useful applications for ion target preparation ${ }^{11}$ and the potential for mass spectrometry schemes. ${ }^{12}$ Ions are stored along a linear trajectory, oscillating back and forth within an electrostatic cavity. Unlike signals acquired from ICRs or Orbitraps, the signal from LEITs

${ }^{a)}$ Electronic mail: j.greenwood@qub.ac.uk. is nonsinusoidal in the time domain. Due to this, the corresponding Fourier transform inherently contains a rich spectrum of harmonics. Through the analysis of these harmonics ${ }^{13}$ LEITs have demonstrated a higher resolution capability than is achievable for a similar trapping time in an Orbitrap. ${ }^{7}$

However, mass analysis of LEIT data is complicated by this existence of multiple harmonics in the Fourier frequency spectra, such that the generation of a mass spectrum is nontrivial, even where there are relatively few ion species.

In this paper an alternative method is proposed for the frequency analysis of anharmonic data acquired from a LEIT. By using comb functions with different time offsets to sample data obtained from several different pickup detectors, and by comparing with a standard fast Fourier transform, a spectrum free of harmonics can be obtained. This CHIMERA (comb-sampling for high-resolution impulse-train frequencyextraction) algorithm allows frequencies to be easily converted into a mass spectrum with substantially higher resolution than can be achieved through Fourier analysis. The implementation of this technique is demonstrated using experimental data obtained from the electrostatic trapping of charged species created by an intense femtosecond laser interaction.

To provide the context for our new analysis approach, we describe our ion trap in Sec. II. A discussion of the Fourier treatment of the signal from the instrument and its drawbacks are given in Sec. III. In Sec. IV the CHIMERA algorithm is presented, while its application to synthetically generated datasets is detailed in Sec. V. The application of 
the CHIMERA algorithm to experimental results is reported in Sec. VI, for both isotope identification in atoms and for molecular fragmentation spectra. In Sec. VII the results are summarized, the performance of the algorithm relative to Fourier analysis is discussed, and suggestions for applications in other devices are made. Appendices are used to detail some of the mathematical derivations.

\section{THE KEIRA ELECTROSTATIC ION TRAP}

The potential energy surface for our kilovolt electrostatic ion reflection analyzer (KEIRA) device is shown in Fig. 1. The electrostatic mirrors at each end of the trap provide axial confinement $(\mathrm{z})$ of the ion motion. The mirror regions consist of six $3 \mathrm{~mm}$ thick plates (R1-R6), with apertures of $16 \mathrm{~mm}$ diameter, separated by $7 \mathrm{~mm}$, and one end plate (R7) situated a further $14 \mathrm{~mm}$ from R6. The potentials that are applied to these electrodes increase incrementally up to a maximum of $5 \mathrm{kV}$.

Radial confinement $(r)$ is provided by two sets of four plates, which are also separated by $7 \mathrm{~mm}$ with $16 \mathrm{~mm}$ diameter apertures. In each set, the outer plates are earthed and the two central plates are held at a negative potential to form an electrostatic lens. Ions are generated by laser ionization of a gas jet, which emerges colinear with the trap axis from a $1 \mathrm{~mm}$ hole in one end plate. The laser is aligned perpendicular to the $\mathrm{z}$ axis, to enter the $14 \mathrm{~mm}$ gap between R6 and R7 and is focused into the gas jet. The ions are generated in a well defined spatial region within a couple of $\mathrm{mm}$ of the trap $z$ axis, with effectively zero initial velocity.

They are immediately accelerated by the electrostatic field into the field free region at the center of the trap. With the appropriate electrode potentials, a large fraction of these ions oscillate between the mirrors on stable trajectories. The ions travel a total distance of approximately $L=410 \mathrm{~mm}$ from mirror to mirror.

KEIRA has three image-charge detectors (pickup rings), one at the geometric center of the trap and one either side, separated by $48 \mathrm{~mm}$ center-to-center. The pickup itself is a cylinder of $16 \mathrm{~mm}$ length and $9 \mathrm{~mm}$ inner diameter (shown in more detail elsewhere ${ }^{15}$ ). It is situated within an earthed housing which has $8 \mathrm{~mm}$ entrance and exit apertures which are separated from the pickup by $1 \mathrm{~mm}$. An ion bunch at the center will have $97 \%$ of its charge imaged on the pickup. ${ }^{15}$

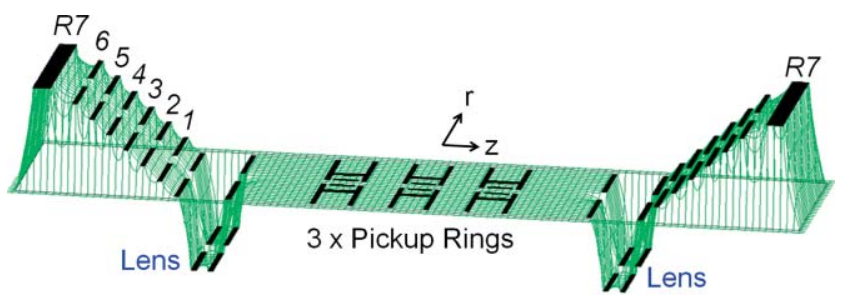

FIG. 1. (Color online) Potential energy surface ${ }^{14}$ for the KEIRA ion trap, with the mirror regions defined by the electrodes R7-R1, and two sets of four electrodes (the inner two held at a negative potential) defining the lenses. Three isolated pickup rings are located in the central, field free region of the trap. The trap is cylindrically symmetric, with ion propagation along the $\mathrm{z}$ axis direction.
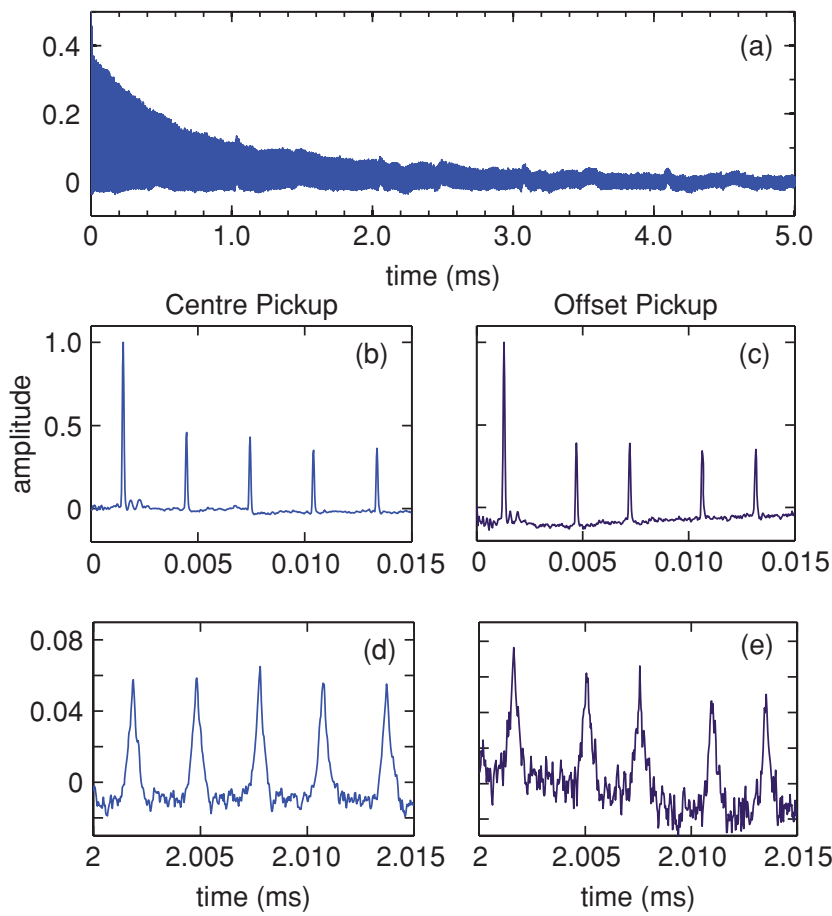

FIG. 2. (Color online) (a) Temporal spectra acquired using the central pickup from ionization of $\mathrm{H}_{2} \mathrm{O}$ present in background gas. (b)-(d) show $15 \mu$ s windows at different trapping times for the central pickup ring (b, d) and a pickup ring closer to the ionization region $(\mathrm{c}, \mathrm{e})$.

Figure 2 shows time signal obtained from photoionization of background gas at a pressure of $4 \times 10^{-8}$ mbar obtained from the central and one offset pickup ring in KEIRA. The ion signal is almost exclusively due to $\mathrm{H}_{2} \mathrm{O}^{+}$, and was produced using the Artemis ${ }^{16}$ femtosecond laser at the Central Laser Facility. The laser pulse had a central wavelength of $790 \mathrm{~nm}$, pulse length of $15 \mathrm{fs}$, and a maximum intensity of about $5 \times 10^{14} \mathrm{~W} \mathrm{~cm}^{-2}$. The trap potentials (in $\mathrm{kV}$ ) were set at $R 1=0.5, R 2=1.0, R 3=1.5, R 4=2.0, R 5=2.5$, $R 6=3.0, R 7=3.55$, Lens $=-2.8$. From a previous calibration of the pickup ring, ${ }^{15}$ we estimate that about $3.2 \times$ $10^{4}$ ions were initially produced by the laser, with $1.5 \times 10^{4}$ being captured on stable trajectories. The data was acquired for $5 \mathrm{~ms}$ and, to improve the statistics, was averaged over $10^{4}$ laser shots. The decay in the amplitude of the signal seen in Fig. 2(a) is due to ion losses from collisions with background gas, and an increase in the bunch length due to slight differences in oscillation times for the ions. In Figs. 2(b) and 2(c) the peak widths correspond to the time taken to traverse the pickup, while the signals at $2 \mathrm{~ms}$ [Figs. 2(d) and 2(e)] indicate that the bunch length now exceeds that of the pickup.

The evolution of ion bunches in LEITs has been extensively studied by the Weizmann group. ${ }^{17}$ They identified three types of behavior corresponding to

$$
\begin{aligned}
\text { coherent diffusion : } t_{w}^{2} & =t_{0}^{2}+n^{2} \Delta T^{2}, \\
\text { non-coherent diffusion : } t_{w}^{2} & =t_{0}^{2}+n \Delta T^{2}, \\
\text { self-bunching : } t_{w}^{2} & =\text { constant, }
\end{aligned}
$$

where $t_{w}$ is the bunch length in time, $t_{0}$ is the initial bunch length, $n$ is the number of full oscillations, and $\Delta T$ is the 
increase in bunch length per oscillation. In coherent diffusion, the ions are weakly perturbed by external or internal (ion-ion) interactions, and differences in ion oscillation times are solely determined by the differences in the initial ion conditions. For noncoherent diffusion, the ion trajectories are disturbed at a rate greater than the oscillation frequency, resulting in the ion having no "memory" of the previous oscillation.

For the data in Fig. 2, the ion density is too low to support the self-bunching phenomenon, but we have observed the self-bunching in KEIRA for larger ion numbers. The present data set exhibits coherent diffusion with $\Delta T \approx 1 \mathrm{~ns}$, a value which can be reduced by tuning the trap potentials.

\section{LEIT MASS RESOLUTION: FOURIER ANALYSIS}

\section{A. Constant ion bunch length}

The mass resolution is defined as $R=m_{0} / \Delta m$, where $m_{0}$ is the ion mass and $\Delta m$ is the minimum resolvable mass difference. We define the following quantities: $f_{0}$ is the ion oscillation frequency, $U$ is the total energy of the ion, $\langle v\rangle$ is the ion velocity averaged over one oscillation, and $L$ is the length of the trap. The frequency of oscillation can be given by

$$
f_{0}=\frac{\langle v\rangle}{2 L}=\frac{\beta}{2 L}\left(\frac{2 U}{m_{0}}\right)^{\frac{1}{2}},
$$

where $\beta$ is a constant that depends on the trap geometry and applied potentials (for KEIRA $\beta \approx 0.75$ ). In terms of frequency, the resolution is given by

$$
R=\frac{m_{0}}{\Delta m} \approx \frac{h f_{0}}{2 \Delta f}
$$

where $\Delta f$ is the full width half maximum (FWHM) of the $h$ th harmonic of the fundamental frequency $f_{0}$.

The signal shown in Fig. 2 can be modeled by a comb function convolved $(\otimes)$ with a distribution function and multiplied by an exponential decay term of lifetime $\tau$. The time distribution generated from one pass of the ion bunch is itself a convolution of the instrumental response of the pickup with the ion density per unit length, which we represent by a Lorentzian with FWHM $t_{w}$, so that the signal in time $(t)$ is given by

$$
\begin{gathered}
s(t) \propto e^{-|t| / \tau} \frac{t_{w}}{t_{w}^{2} / 4+t^{2}} \otimes\left\{\sum_{n=-\infty}^{\infty} \delta\left(t-\frac{(n+c)}{f_{0}}\right)\right. \\
\left.+\delta\left(t-\frac{\left(n+\frac{1}{2}-c\right)}{f_{0}}\right)\right\},
\end{gathered}
$$

where $c / f_{0}$ is the time taken for the ions to travel from the geometric center of the trap to the center of the pickup used to acquire the data. We formally consider a signal symmetric in time, for mathematical convenience. The values of $c$ for the three pickups in KEIRA are approximately 0 and \pm 0.038 , and change only slightly for different potential energy surfaces. If $\Delta T \approx 0, t_{w}$ remains constant and the amplitude of the Fourier
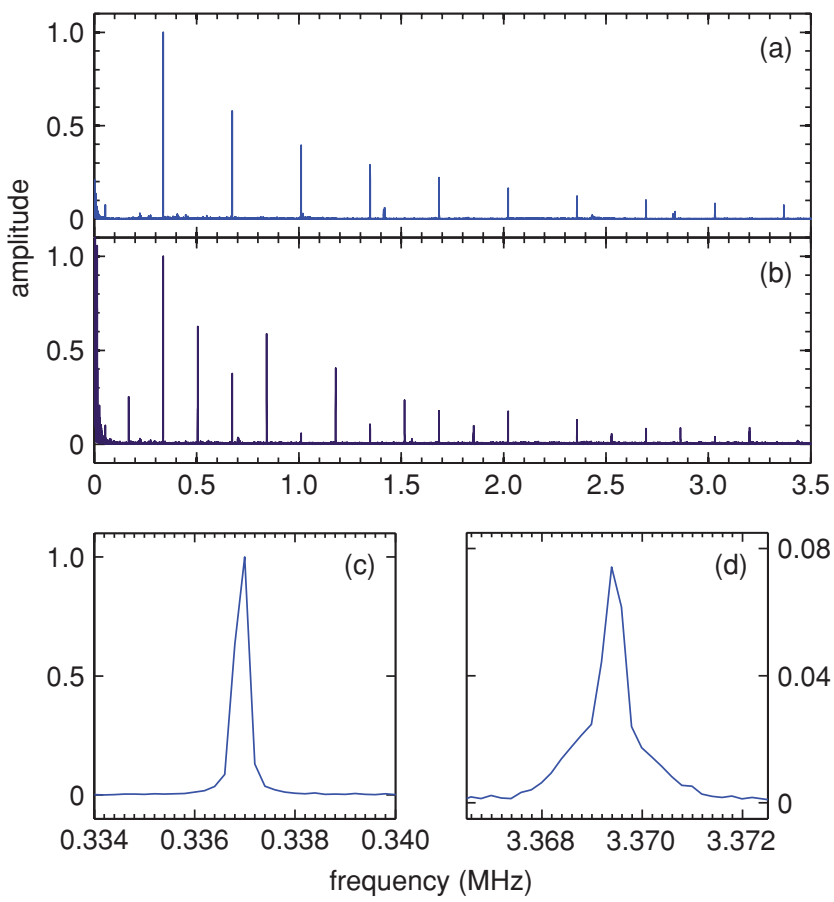

FIG. 3. (Color online) FFT with a Welch window of the data in Fig. 2 for the (a) central and (b) offset pickups, with closer inspection of (c) the 2nd and (d) 20th harmonics for the central pickup only.

transform for positive frequencies $(f)$ is

$$
\begin{aligned}
|S(f)| \propto & e^{-\pi f t_{w}}\left|\cos \frac{\pi f\left(2 c-\frac{1}{2}\right)}{f_{0}}\right| \\
& \times \sum_{h=1}^{\infty} \frac{\tau^{-1}}{\tau^{-2}+\left(2 \pi\left(f-h f_{0}\right)\right)^{2}}
\end{aligned}
$$

(see Appendix A). Figure 3 shows the Fourier transforms of the two pickup signals from Fig. 2. For harmonic frequencies $f \approx h f_{0}$ and a central pickup with $c=0$, Eq. (5) reduces to

$$
|S(f)| \propto \tau\left(e^{-\pi h f_{0} t_{w}}\right)\left(1+(-1)^{h}\right)^{1 / 2} .
$$

Therefore, in Fig. 3(a) for the central pickup, the odd harmonics are suppressed, while the even ones reduce exponentially at a rate determined by $t_{w}$. For the offset pickup [Fig. 3(b)], odd harmonics are included and the magnitudes follow the more complex formula given in Eq. (5). Note that the 13th harmonic is absent since for $c=-0.038,2 \pi f(1 / 2-2 c) / f_{0}$ $\approx 15 \pi$.

From Eq. (6), the FWHM of the harmonic peaks $\left(\Delta f_{\tau}\right.$ $=1 / \tau \pi)$ is independent of $h$, hence the resolution increases linearly with the harmonic order

$$
R=\frac{\pi}{2} h f_{0} \tau
$$

This has been exploited to achieve the very high resolutions previously attained for a LEIT in self-bunching mode. ${ }^{12}$

\section{B. Coherent bunch diffusion}

Figures 3(c) and 3(d) show the 2nd and 20th harmonics from the center pickup data. The second harmonic represents 
a resolution of $R \approx 500$. According to Eq. (3), $\Delta f$ should be the same for both harmonics, but due to bunch diffusion the 20th harmonic is broader. If the ion bunch diffuses coherently [Eq. (1)], and we assume that the initial bunch width $t_{0}$ is negligible, then $t_{w}=n \Delta T$ in Eq. (4). The Fourier transform for this signal is derived in Appendix B. For frequencies close to the $h$ th harmonic $f=h f_{0}+\delta f$ and for $c=0, \tau \rightarrow \infty, h$ even, we obtain

$$
|S(\delta f)| \propto\left(\frac{1}{\left(1-e^{-\pi f \Delta T}\right)^{2}+e^{-\pi f \Delta T}\left(2 \pi \delta f / f_{0}\right)^{2}}\right)^{1 / 2},
$$

which for low bunch spread per oscillation, i.e., $f_{0} \Delta T \ll 1$, yields

$$
|S(\delta f)| \propto\left(\frac{1}{\left(\pi h f_{0} \Delta T\right)^{2}+\left(2 \pi \delta f / f_{0}\right)^{2}}\right)^{1 / 2} .
$$

In this case, the contribution to the FWHM of the harmonic due to coherent bunch dispersion is

$$
\Delta f_{\Delta T}=\sqrt{3} h f_{0}^{2} \Delta T .
$$

Therefore if the ion lifetime is long, the peak width increases linearly with $h$ and hence there is no improvement in resolution for higher harmonics. In Figs. 3(c) and 3(d) it can be seen that the 20th harmonic is wider than that of the 2nd. It is also evident from Fig. 2 that a proportion of the ions disperse more slowly than the rest, resulting in the multiple component peak in Fig. 3(d).

\section{Windowing and discretization}

In practice, the acquisition time $T_{S}$ is finite $(5 \mathrm{~ms}$ in Fig. 2) and the signal is multiplied by an apodization or windowing function $a\left(t / T_{S}\right)$ to reduce spectral leakage in the Fourier transform. Therefore $S(f)$ is also convolved with the Fourier transform of $a\left(t / T_{S}\right)$, which introduces additional broadening to harmonic peaks so that

$$
(\Delta f)^{2} \approx\left(\Delta f_{\tau}\right)^{2}+\left(\Delta f_{\Delta T}\right)^{2}+\left(\Delta f_{A}\right)^{2},
$$

where $\Delta f_{A}=A / T_{S}$ and $A$ is a constant specific to the apodization function, e.g., for a rectangle window $A=1.21$; Welch $A=1.59$; Blackman $A=2.3$. With a Welch window it is only for $T_{S}>5 \tau$ that $\Delta f_{A}<\Delta f_{\tau}$. Therefore, the peak width of the 2nd harmonic in Fig. 3(c) is dominated by the apodization $\Delta f_{A}=318 \mathrm{~Hz}$.

As the data is also discrete, the Fast Fourier Transform (FFT) calculates the frequency spectrum at $T_{S}^{-1}$ intervals (200 Hz), which is often similar to $\Delta f$ [Fig. 3(c)]. This can be mitigated by zero padding the data, but the discontinuity this introduces at $t=T_{S}$ can result in additional spectral leakage.

\section{Harmonic phase}

Since the ion bunch has a well defined starting point in time, in principle there is a clear phase associated with Fourier components from ion trapping signal, which should allow discrimination against uncorrelated electronic noise. If $\gamma / f_{0}$ is the time taken by the ion bunch to reach the geometric center of the trap after its initial formation ( $\gamma=0.25$ for KEIRA), the phase of the Fourier frequencies is given by

$$
\phi(f)=-2 \pi \frac{f}{f_{0}}\left(\gamma+\frac{1}{4}\right)
$$

(see Appendix C). In principle, there is additional information encoded in the phase which could be used to identify the harmonic order and to distinguish signal from external noise sources. However, this is very difficult to achieve in practice as the phase changes rapidly across the finite width of a harmonic peak.

\section{E. Comparison with a time of flight mass spectrometer}

Some LEITs have been employed as multi-reflectron time of flight mass spectrometers, where ions are trapped for long enough to achieve mass separation and then extracted. ${ }^{18}$ By significantly increasing the effective time of flight length, large enhancements in resolution capabilities can be achieved compared to a standard linear or single-reflection device. However, such a device is limited to studying similar mass ions to ensure different ion bunches undergo the same number of oscillations.

If an ion bunch of width $t_{w}$ is extracted from a LEIT after a time $T_{S}$, the time of flight resolution is $T_{S} / 2 t_{w}$. To compare with the resolution achievable using Fourier analysis, consider ions trapped for the same time with $t_{w} f_{0}$ $=0.05$ and $\tau \rightarrow \infty$. Using a Welch window, an equivalent resolution would only be possible from the 32nd harmonic or greater.

\section{F. Drawbacks of Fourier analysis}

The signal obtained from LEITs is nonsinusoidal unlike those obtained from other mass spectrometers such as an ICR or an Orbitrap. While higher harmonic components give better resolution, there are a number of drawbacks in using Fourier transforms to analyze this signal;

(1) The spectral amplitude of the signal is spread out among many harmonics, reducing the signal-to-noise ratio, particularly for higher harmonics.

(2) The presence of multiple harmonics makes conversion of frequencies into a mass spectrum difficult, especially if many different ions are present.

(3) As it is possible to have many pickup detectors acquiring data simultaneously, one would expect that these different channels could be combined to yield more information and better signal-to-noise, but the spectra from pickups offset from the trap center generate even more complicated harmonic spectra.

(4) There is rich phase information present in the signal which could be used to discriminate against electronic noise and improve resolution, but this is very difficult to extract from the Fourier transform. 
(5) Apodization of the data is necessary to reduce spectral leakage, but this reduces the achievable resolution and the signal-to-noise ratio.

\section{CHIMERA COMB-SAMPLING THEORY}

\section{A. Determining the frequency of a comb}

Consider a time signal $s(t)$ which consists of a series of impulses at regular intervals,

$$
s(t)=\sum_{n=0}^{N} \delta\left(t-\frac{n}{f_{0}}\right) .
$$

In this case, we use $\delta$ to represent a narrow function of finite height. The frequency of such a distribution can be determined from a simple measurement, that is, to simply count the time between one impulse and the next. For such a scheme, the resolution increases as the number of impulses measured is increased. However, executing this measurement becomes nontrivial if the signal contains additional frequencies. Alternatively, using a comb function $g(t)$, a spectral function $\mathcal{S}(f)$ may be generated, which gives the desired frequency $f_{0}$,

$$
\begin{gathered}
g(t)=\sum_{m=m_{1}}^{m_{2}} \delta\left(t-\frac{m}{f}\right), \\
\mathcal{S}(f)=\frac{1}{M} \int_{0}^{\infty} s(t) g(t) d t,
\end{gathered}
$$

where $M=m_{2}-m_{1}+1$. However, $\mathcal{S}(f)$ would also contain an infinite number of harmonics and "fractional harmonics."

Introducing an offset from $t=0$, such that both $s(t)$ and $g(t)$ are temporally offset by an amount $\gamma / f_{0}$, gives

$$
\begin{aligned}
\mathcal{S}(f)= & \frac{1}{M} \int_{0}^{\infty}\left[\sum_{n=0}^{N} \delta\left(t-\frac{(n+\gamma)}{f_{0}}\right)\right. \\
& \left.\times \sum_{m=m_{1}}^{m_{2}} \delta\left(t-\frac{(m+\gamma)}{f}\right)\right] d t .
\end{aligned}
$$

In this case, more than one coincidence of the impulses only occurs if

$$
\frac{f}{f_{0}}=\frac{m+\gamma}{n+\gamma} .
$$

Therefore if $\gamma$ is chosen to be irrational, $\mathcal{S}(f)$ only has a significant value when $f=f_{0}$. LEITs are normally filled with ions by injection of a bunch from outside the trap, where bunches can be generated by, for example, chopping a continuous beam, pulsing ions out of a pretrap, or ionization with a short pulse laser. For ions which emanate from such a pulsed ion source and subsequently execute trajectories dictated by electrostatic optics, the value of $\gamma$ will be constant, irrespective of the ion mass.

In practice, the measured impulses from an ion bunch passing through the pickup are finite in time, so that there will still be contributions from fractional harmonics even if $\gamma$ is an irrational number. If a second pickup is used to acquire data simultaneously, it will have a different value of $\gamma$ as the distance between ion source/pulsar and pickup has changed. Analysis of this data for the new $\gamma$ will also have fractional harmonics but at different values to the signal from the first pickup. Therefore, by multiplying the two spectra and taking the square root, the fractional harmonics will be suppressed, producing a frequency spectrum with the main contribution being from the fundamental frequencies only.

\section{B. Resolution}

As a comb function is a better match to the pickup signal than a sinusoid, one would expect the frequency resolution to be significantly improved. To simulate a more realistic signal, we convolve the comb function with a pickup response function $q(t)$. For mathematical convenience, we will approximate $q(t)$ by a triangle function of full width in time $2 t_{w}$ and amplitude $t_{w}^{-1}$, such that

$$
\begin{aligned}
& q(t)=\frac{1}{t_{w}}\left(1-\frac{|t|}{t_{w}}\right), \quad|t| \leq t_{w}, \\
& q(t)=0, \quad|t|>t_{w} .
\end{aligned}
$$

Convolving this with the comb function in Eq. (13), we may express the signal as

$$
s(t)=\sum_{n=0}^{N} q\left(t-\frac{n}{f_{0}}\right)
$$

(where we have set $\gamma=0$ ). The corresponding frequency function [cf. Eq. (16)] is thus

$$
\begin{aligned}
\mathcal{S}(f) & =\frac{1}{M} \int_{0}^{\infty} \sum_{n=0}^{N} q\left(t-\frac{n}{f_{0}}\right) \sum_{m=m_{1}}^{m_{2}} \delta\left(t-\frac{m}{f}\right) d t, \\
& =\frac{1}{M} \sum_{n=0}^{N} \sum_{m=m_{1}}^{m_{2}} q\left(\frac{m}{f}-\frac{n}{f_{0}}\right) .
\end{aligned}
$$

Now, with $t_{w}$ as the FWHM of $q(t)$, where $t_{w} \ll 1 / f_{0}$, and if we let $f=f_{0}+\delta f$ where $\frac{\delta f}{f_{0}}<1 / m$, there are contributions to $\mathcal{S}(f)$ only when $m=n$, giving

$$
\mathcal{S}(\delta f)=\frac{1}{M} \sum_{m=m_{1}}^{m_{2}} q\left(\frac{m \delta f}{f_{0}^{2}}\right) .
$$

To model the effect of coherent diffusion, we let

$$
t_{w}=(k+m p) / f_{0},
$$

where $k / f_{0}$ is the initial FWHM, and the increase in width per oscillation is $\Delta T=p / f_{0}$, giving

$$
\mathcal{S}(\delta f)=\frac{f_{0}}{M} \sum_{m=m_{1}}^{m_{2}}\left(\frac{k+p m-m \frac{|\delta f|}{f_{0}}}{(k+p m)^{2}}\right) .
$$

For no bunch diffusion, $p=0$, and for small values of $\delta f$ this yields

$$
\mathcal{S}(\delta f)=\frac{f_{0}}{k}\left(1-\frac{|\delta f|}{2 f_{0} k}\left(m_{2}+m_{1}\right)\right) .
$$




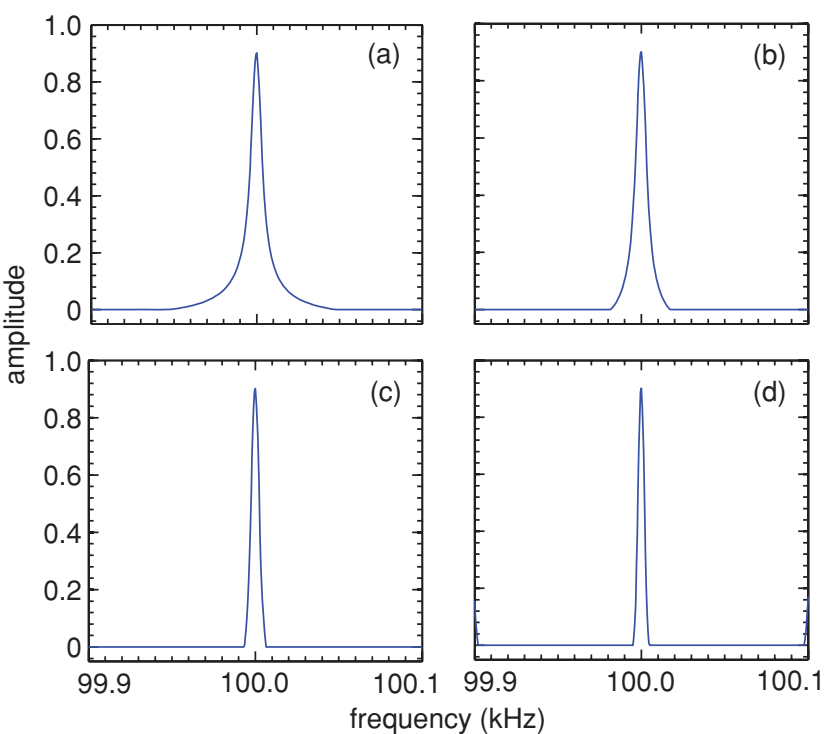

FIG. 4. (Color online) Comb-sampling frequency analysis for simulated data with a frequency of $100 \mathrm{kHz}$ and FWHM of $200 \mathrm{~ns}$ for analysis windows now extending from (a) $0-5$, (b) $0.5-5$, (c) $2.5-5$, and (d) $4.5-5 \mathrm{~ms}$.

(see Appendix D). This is also a triangle function, giving resolution $R$,

$$
R=\frac{f_{0}}{2 \Delta f}=\frac{m_{2}+m_{1}}{4 k} .
$$

It is worth noting that as the sampling window narrows toward the end of the time data, i.e., $m_{2}=N, m_{1}$ $\rightarrow m_{2}$, where $N=f_{0} T_{S}$ is the number of oscillations within the sample time, the resolution for a time of flight instrument is recovered,

$$
R \rightarrow \frac{m_{2}}{2 k}=\frac{T_{S}}{2 t_{w}} .
$$

At the other extreme, if the sampling window spans the whole data set $\left(m_{1}=0, m_{2}=N\right)$, the resolution is a factor of two lower. In Fig. 4, synthetic data with parameters $f_{0}=100 \mathrm{kHz}$, $T_{S}=5 \mathrm{~ms}, N=1000, t_{w}=200 \mathrm{~ns}, k=0.04$, and $p=0$ has been analyzed using comb-sampling for four sample windows with different starting points $\left(m_{1} / f_{0}=0,0.5,2.5,4.5 \mathrm{~ms}\right)$ and the same end point $\left(m_{2} / f_{0}=5 \mathrm{~ms}\right)$. Note that the mean value of the whole synthetic data set has been subtracted from each data point prior to analysis.

As predicted from Eq. (25), delaying the start of the sampling increases the resolution, so that in Fig. 4(d) the resolution is close to that expected for a conventional TOF spectrometer with a $5 \mathrm{~ms}$ flight time. Figure 5 shows the same data but focusing on amplitudes close to zero and over a greater range of frequencies. On either side of the main peak the frequency function dips below zero. This corresponds to frequencies where the comb "misses" the pickup impulses and samples only the baseline $(=-$ mean). There is also a ringing artifact which begins when the sampling frequency is far enough away from $f_{0}$ to start sampling the next impulse at the end of the time window when

$$
N f_{0}=m_{2} f=(N \pm 1) f,
$$

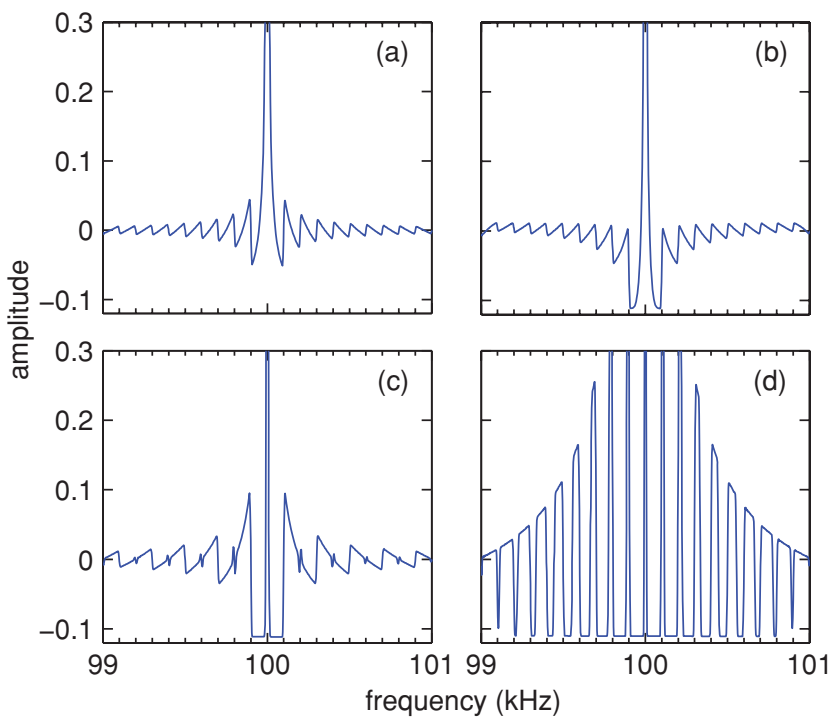

FIG. 5. (Color online) As for Fig. 4 but with the frequency range increased and a reduced amplitude range.

$$
f-f_{0}= \pm \frac{f}{N}= \pm 100 \mathrm{~Hz} .
$$

In Fig. 5(d) the ringing artifact is unacceptably high since a number of different frequencies are capable of sampling most of the impulses in this small sample window. The best outcome can be found for Fig. 5(b) where the start time of the window is $10 \%$ of the end time. Here, nearly all of the ringing is confined to negative values which can be eliminated if values less than zero are rejected. Delaying the start of the sampling also ensures that for real data, no ions on unstable trajectories contribute to the frequency spectrum.

Alternatively, coherent bunch diffusion with $k=0$, yields

$$
\mathcal{S}(\delta f)=\frac{f_{0}}{M p}\left(1-\frac{|\delta f|}{p f_{0}}\right) \ln \left(\frac{m_{2}}{m_{1}}\right), \quad \text { if } m_{1}, m_{2} \gg 1
$$

(see Appendix D), giving a resolution of $R=1 / 2 p$. In this case, the resolution is solely determined by the rate at which the bunch diffuses and not upon the length of the acquisition time.

\section{Influence of the ion lifetime and windowing}

To simulate the influence of the ion lifetime $\tau$, the data is given a weighting which linearly decreases in time with a gradient of $\tau^{-1}$, so that $q(t)$ now becomes

$$
q(t)=\frac{1}{t_{w}}\left(1-\frac{|t|}{t_{w}}\right)\left(1-\frac{m}{\tau f_{0}}\right), \quad|t| \leq t_{w},
$$

effectively creating a trapezoidal window for the data. Taking the case of zero bunch diffusion $(p=0)$ and $m_{1}=0$, the resolution becomes

$$
R=\frac{m_{2}\left(3 \tau f_{0}-2 m_{2}\right)}{6 k\left(2 \tau f_{0}-m_{2}\right)}
$$


(see Appendix E). This reduces to Eq. (25) as $\tau \rightarrow \infty$ and if the lifetime is equal to the width of the analysis window ( $\tau$ $\left.=m_{2} / f_{0}\right)$, the resolution is a factor of $1.5 \operatorname{lower}\left(R=m_{2} / 6 k\right)$.

Another advantage of this technique, compared to FFT, is that any shape of window can be employed. Since the analysis is more sensitive to impulse peaks near the end of the time spectrum, their relative weight can be enhanced. For instance, a simple linear weighting increases the resolution by a factor of $4 / 3\left(R=m_{2} / 3 k\right.$, Appendix E).

\section{IMPLEMENTATION OF CHIMERA ALGORITHM}

In this section, we extend the analysis to the general case and apply it for the setup currently employed in KEIRA. If we include the pickup offset, $c$, then Eqs. (13) and (14) now become

$$
\begin{aligned}
s(t)= & \sum_{n=0}^{N}\left\{\delta\left(t-\frac{(n+\gamma+c)}{f_{0}}\right)\right. \\
& \left.+\delta\left(t-\frac{\left(n+\gamma+\frac{1}{2}-c\right)}{f_{0}}\right)\right\}, \\
g(t)= & \sum_{m=m_{1}}^{m_{2}}\left\{\delta\left(t-\frac{(m+\gamma+c)}{f}\right)\right. \\
& \left.+\delta\left(t-\frac{\left(m+\gamma+\frac{1}{2}-c\right)}{f}\right)\right\} .
\end{aligned}
$$

For a proportion of the teeth in these two combs to coincide periodically, i.e., contribute to $\mathcal{S}(f)$ more than once, one of the following conditions must be met:

$$
\begin{aligned}
\frac{f}{f_{0}} & =\frac{m+\gamma+c}{n+\gamma+c}, & \frac{f}{f_{0}} & =\frac{m+\gamma+\frac{1}{2}-c}{n+\gamma+c}, \\
\frac{f}{f_{0}} & =\frac{m+\gamma+\frac{1}{2}-c}{n+\gamma+\frac{1}{2}-c}, & \frac{f}{f_{0}} & =\frac{m+\gamma+c}{n+\gamma+\frac{1}{2}-c},
\end{aligned}
$$

where $m, n$ are integers in the specified ranges. The magnitude of $\mathcal{S}(f)$ is determined by the fraction of the sample comb data which yields coincidences normalized to the value at $f=f_{0}$. Theoretical spectra for a pure comb signal with similar conditions to those used in KEIRA $(\gamma=1 / 4, c=0$ and $\gamma=1 / 4, c=-1 / 28)$ are shown in Fig. 6 . For the center pickup $(c=0)$, it can be seen that the relative contribution of some fractional harmonics is high, particularly for $f / f_{0}=1 / h$ where $h$ is odd. The even harmonics are absent while odd harmonics are reduced in amplitude by a factor of $h$. In contrast, for the offset pickup fractional harmonics are significantly reduced in magnitude, with no contribution at integer values.

As the frequency peaks in Figs. 6(a) and 6(b) only coincide for $f=f_{0}$, when the two spectra are multiplied together only the fundamental remains. For real data, the finite width of the image charge impulses does result in some contributions at other frequencies giving an incomplete suppression of fractional harmonics. In this case, acquiring an additional

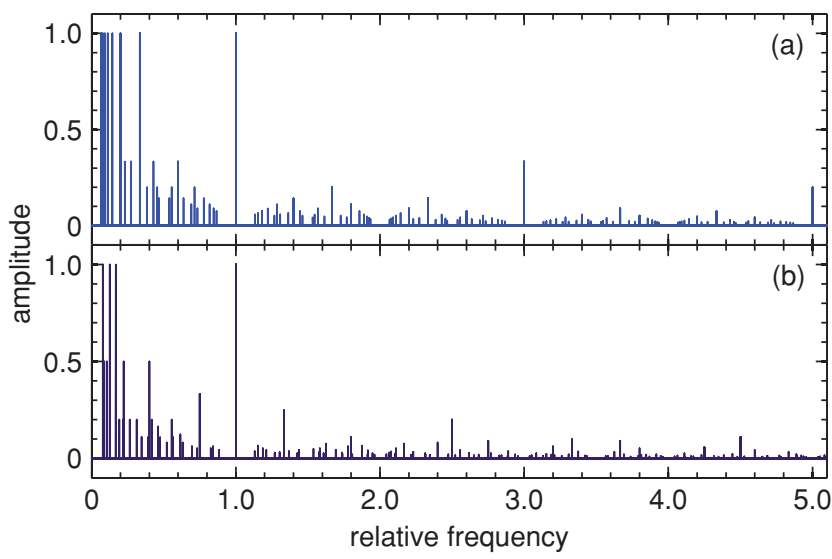

FIG. 6. (Color online) Spectrum from analysis of a pure data comb at frequency $f_{0}$ for (a) central pickup ring with $\gamma=1 / 4, c=0$, and (b) offset pickup ring with $\gamma=1 / 4, c=-1 / 28$ (approximately equal to the experimental value of -0.038 )

spectrum from a third pickup enables fractional harmonics to be further suppressed by multiplying all three together and taking the cube root.

In practice, the teeth of the comb are given a finite width so that more than one data point is sampled per peak. The average of all these data points is then normalized by the number of teeth in the comb $M$. Increasing the width of the teeth reduces the achievable resolution, but the change is negligible provided that the width remains less than $t_{w}$. If ions of different mass have the same bunch length in space, then $t_{w} \propto m_{0}^{1 / 2} \propto f_{0}^{-1}$. Therefore, for different sampling frequencies $f$, the algorithm changes the teeth width in proportion to $f^{-1}$.

Figure 7 shows CHIMERA sampling analysis for simulated data generated using the same parameters as those used in Fig. 4, for the frequency range $0.1 f_{0}-5.1 f_{0}$. Data for three pickups $(c=0,-0.038,+0.06)$ has been generated [Figs. 7(a), 7(b), and 7(c), respectively]. Also shown are the results of combining two of the spectra $[c=0,-0.038$, Fig. 7(d)] and all three [Fig. 7(e)] together. It can be seen that there is a steady improvement in the purity of the spectrum as the number of combined data sets increases. In principle this will improve further if data from additional pickups is included.

To achieve a still purer spectrum, the FFT can be used as a discriminator. Comparing a comb-sampling spectrum with the FFT for $c=0$ (at double the frequency since odd harmonics are suppressed), if the FFT lies below a user set discrimination level, the contribution to the comb-sampling spectra may be set to zero at that frequency.

\section{ANALYSIS OF KEIRA RESULTS}

So far we have applied the CHIMERA algorithm only to synthetic data. Figure 8 shows the analysis of real data acquired for $T_{S}=8 \mathrm{~ms}$ from ionization of Xe gas by $40 \mathrm{fs}$ laser pulses with a maximum intensity of $2.5 \times 10^{13} \mathrm{~W} \mathrm{~cm}^{-2}$, averaged over 4000 shots. In Fig. 8(a), the FFT of data from the center pickup shows 14 even harmonics above the general noise level. In Fig. 8(b), the comb-sampling method has 


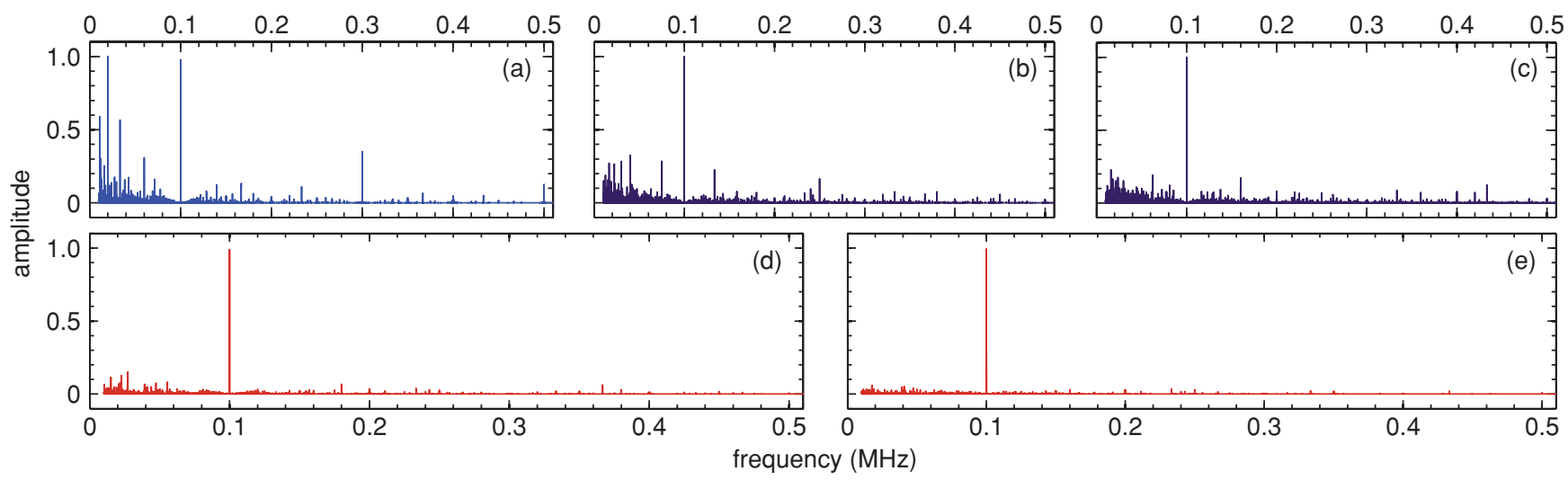

FIG. 7. (Color online) Spectra from analysis of simulated data for a single ion species oscillating at a frequency of $100 \mathrm{kHz}$ with $\gamma=1 / 4$ and (a) $c=0$, (b) $c=-0.038$, (c) $c=+0.06$, (d) $c=0,-0.038$ combined, (e) all combined.

been applied using a window from $0.8-8 \mathrm{~ms}$ for both center and offset pickup data, with a discriminator set just above the general FFT noise in Fig. 8(a). Apart from a few minor contributions, harmonics are absent from the comb analyzed spectrum. The frequency spectrum for isotopes of $\mathrm{Xe}^{+}$is shown in Fig. 8(c), where the second harmonic of the FFT is compared with CHIMERA analysis, which gives a resolving power of 5000, a factor 15 greater than the second harmonic in the FFT. Whilst an equivalent FFT resolution can be obtained from the 30th harmonic as predicted by Eq. (3), this peak is barely discernable above the noise level.

It can be seen that the relative amplitudes of the xenon isotopes obtained from FFT and CHIMERA are different. While the results of the CHIMERA analysis are in good agreement with natural abundances of these isotopes, the agreement for the FFT is poor. This is probably due to the limitation on the FFT frequency step size as discussed in Sec. III C.

As CHIMERA removes harmonics, it is also straightforward to generate a mass spectrum. To demonstrate this, an experiment was carried out on a gaseous target of $\alpha$-Cyano4-hydroxycinnamic acid (CHCA), which has a formula of $\mathrm{C}_{10} \mathrm{H}_{7} \mathrm{NO}_{3}$ and a molecular mass of 189.17 . The neutral target of this molecule was produced by depositing a sample of CHCA onto the surface of the final electrode, which was then desorbed off the surface using a $4 \mathrm{~ns}, 355 \mathrm{~nm}$ laser pulse. Subsequent ionization/fragmentation was enforced by a $40 \mathrm{fs}$ laser pulse of intensity $10^{13} \mathrm{~W} \mathrm{~cm}^{-2}$. Data was acquired for $3 \mathrm{~ms}$ using the central pickup and one offset pickup detector. Figure 9 compares mass spectra produced from a FFT of the central pickup signal and from the comb-sampling analysis using data from both pickups. As well as the improved

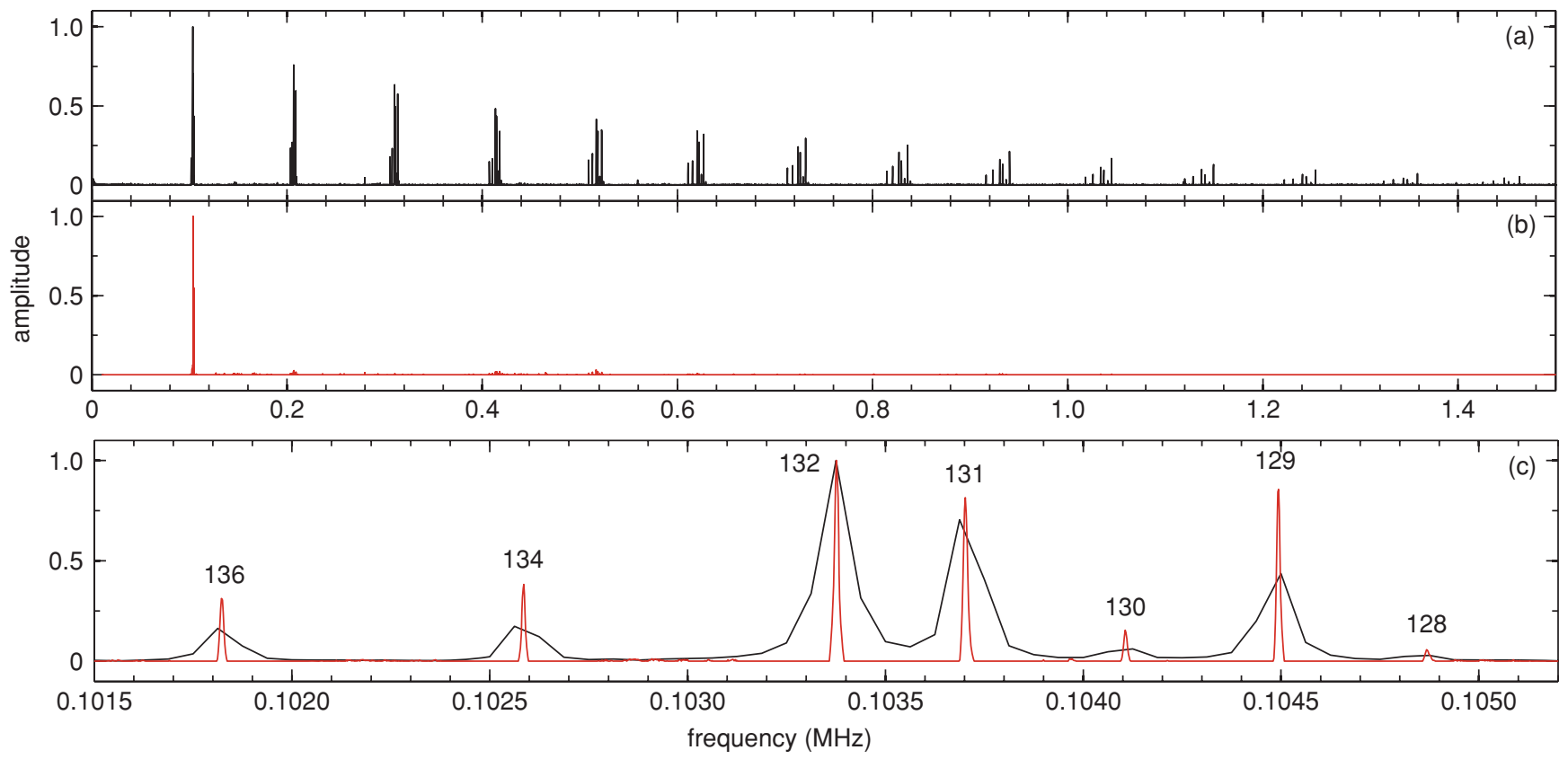

FIG. 8. (Color online) Frequency analysis of $\mathrm{Xe}^{+}$isotopes trapped for $8 \mathrm{~ms}$ using (a) FFT with a Welch window and (b) comb-sampling with a window from 0.8-8 ms. Plot (c) shows the fundamental frequency from comb-sampling (narrow peaks) compared to the second harmonic of the FFT. Corresponding masses of Xe isotopes are indicated. 


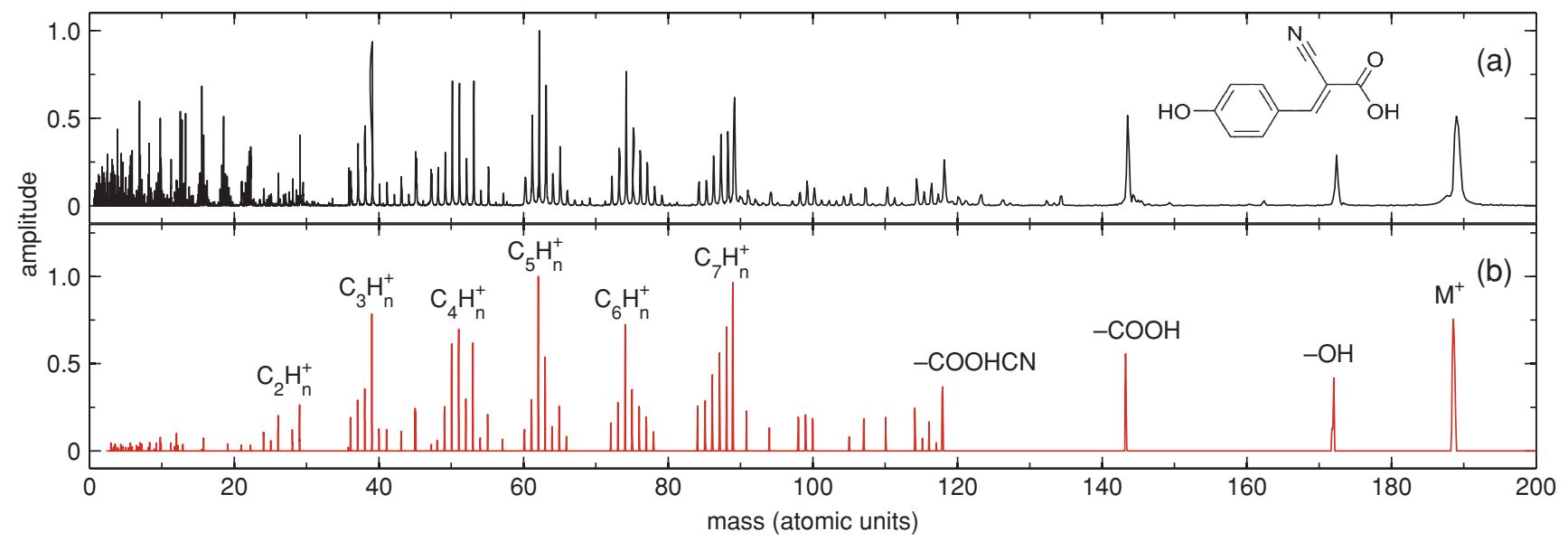

FIG. 9. (Color online) Frequency analysis of ions generated from ionization of CHCA trapped for $3 \mathrm{~ms}$ from (a) FFT of the center pickup data with a Welch window, and (b) comb-sampling of data from the center pickup and the offset pickup closest to the femtosecond laser focus. $\mathrm{M}^{+}$is the parent ion, with other fragments corresponding to the hydrocarbons shown, or to the loss (-) of a particular chemical group.

resolution of the comb-sampling method, it can be seen that multiple harmonics in Fig. 9(a) make analysis of masses less than 30 very difficult with FFT. For low masses in Fig. 9(b) there are some fractional harmonics present, but these are at a low level and could be further suppressed if data from additional pickups was available.

\section{SUMMARY}

In this paper, a new algorithm (CHIMERA) for analyzing the oscillation frequencies of ions in a linear electrostatic trap has been described. By using a comb function to sample the data at different frequencies, it has been shown that more complete utilization of the information contained in the ion signal is possible. The key to extracting only the fundamental frequency is for the initial ion bunch generation to be temporally and spatially well defined. By generating multiple spectra using data acquired from more than one pickup detector, integer and fractional harmonics arising from the finite width of the pickup impulses can be suppressed.

We have tested CHIMERA on simulated data and real data acquired from a linear electrostatic ion trap using in situ ion generation with a femtosecond laser. As the sampling of the narrow pickup peaks is very sensitive to the frequency of the comb, mass resolutions obtained from relatively short trapping periods $(<10 \mathrm{~ms})$ are shown to approach those which could be obtained for a linear time of flight device of equivalent length (about $100 \mathrm{~m}$ ). For instance, a mass resolution of 5000 was obtained for $\mathrm{Xe}^{+}$isotopes for a trapping time of $8 \mathrm{~ms}$, which was a 15 fold improvement over the second harmonic obtained from a Fourier transform. CHIMERA also benefits in that it does not suffer from spectral leakage, which is a feature of the windowing process in a Fourier transform. And unlike FFT algorithms, there are no limitations on the frequency steps used.

The value of the initial offset $(\gamma)$ of the comb data and that of the pickup electrodes from the trap center $(c)$, in the present setup have been fixed by the existing geometry and operational mode $(\gamma=1 / 4, c=0,-0.038)$. While ideally these values should be irrational, this is not possible in practice due to the finite width of the recorded impulses. However, if $\gamma$ and $c$ are chosen to avoid values close to factorized fractions with small denominators, significant contributions from fractional harmonics can be avoided.

As such our present value of $\gamma=1 / 4$ is not ideal, but could be changed by pulsing the potential of the end plate R7 once all the ions have first left the ion generation region. For instance, if $\mathrm{R} 7=3.2 \mathrm{kV}$ when the ions are created and is raised to $3.55 \mathrm{kV}$ once the ions reach the field free region, a new $\gamma$ value of 0.283 would be generated. With appropriate choices of $\gamma$ and $c$, CHIMERA could also be easily applied to other electrostatic traps and rings for which ion bunches are injected from an external source. For instance, ion bunches from a matrix-assisted laser desorption and ionization source ${ }^{19}$ and a pulsed beam ${ }^{20}$ have previously been injected into this type of trap using electrostatic optics. Another possible implementation would be for the case where ions are confined and cooled in a radiofrequency trap prior to injection into a storage ring, provided the ions are injected directly rather than being mass selected by a magnet. ${ }^{21}$

Application to other mass spectrometers, such as the Orbitrap and ICR may be possible if highly anharmonic signals are acquired. The potential for generating higher mass resolution from anharmonic signals in an ICR has previously been recognised. ${ }^{22,23}$ For some pickup electrode arrangements and ion excitation schemes, signals from multiple pickups or the differential signal from pairs could exhibit a signal resembling the pulse trains analyzed in this paper. ${ }^{24}$

In conclusion, the ability to extract high resolution mass spectra from a linear electrostatic ion trap has been enhanced by employing a new CHIMERA analysis method, which samples ion oscillation data with a comb function. Compared with Fourier analysis, the CHIMERA algorithm makes better use of the phase and frequency information present in the data. This enables the fundamental ion oscillation frequencies to be extracted with much higher resolution, while also suppressing uncorrelated electronic noise. When this algorithm is combined with the mass independent trapping and ion detection characteristics of the electrostatic trap, very complex ion 
mixtures with an enormous mass range can be simultaneously analyzed with very high resolution.

\section{ACKNOWLEDGMENTS}

The authors wish to note with appreciation, insightful discussions with Gleb Gribakin who provided valuable input to this paper. This work was supported by Leverhulme Trust and utilized the Artemis Laser at the STFC Central Laser Facility. C.R.C. and O.K. acknowledge funding from the Leverhulme Trust. L.B., M.J.D., and R.B.K. acknowledge funding from Department of Employment and Learning (Northern Ireland) and J.D.A. acknowledges funding from the European Social Fund.

\section{APPENDIX A: FOURIER TRANSFORM FOR AN ION BUNCH OF CONSTANT LENGTH}

$$
\begin{aligned}
& s(t) \propto e^{-|t| / \tau} \frac{t_{w}}{t_{w}^{2} / 4+t^{2}} \otimes\left(\sum_{n=-\infty}^{\infty} \delta\left(t-\frac{(n+c)}{f_{0}}\right)\right. \\
& \left.+\delta\left(t-\frac{\left(n+\frac{1}{2}-c\right)}{f_{0}}\right)\right),
\end{aligned}
$$

for this signal with Lorentzian peaks and constant width $t_{w}$, the Fourier transform is

$$
\begin{aligned}
S(f) & \propto \frac{\tau^{-1}}{\tau^{-2}+(2 \pi f)^{2}} \\
& \otimes\left(e^{-\pi|f| t_{w}}\left(\sum_{n=-\infty}^{\infty} e^{-\frac{2 \pi i f(n+c)}{f_{0}}}+e^{-\frac{2 \pi i f\left(n+\frac{1}{2}-c\right)}{f_{0}}}\right)\right), \\
S(f) & \propto \frac{\tau^{-1}}{\tau^{-2}+(2 \pi f)^{2}} \\
& \otimes\left(e^{-\pi|f| t_{w}}\left(1+e^{\frac{2 \pi i f\left(2 c-\frac{1}{2}\right)}{f_{0}}}\right) e^{-\frac{2 \pi i f c}{f_{0}}} \sum_{n=-\infty}^{\infty} e^{-\frac{2 \pi i f n}{f_{0}}}\right),
\end{aligned}
$$

$$
\begin{aligned}
S(f) & \propto \frac{\tau^{-1}}{\tau^{-2}+(2 \pi f)^{2}} \\
& \otimes\left(e^{-\pi|f| t_{w}}\left(1+e^{\frac{2 \pi i f\left(2 c-\frac{1}{2}\right)}{f_{0}}}\right) e^{-\frac{2 \pi i f c}{f_{0}}} \sum_{h=-\infty}^{\infty} \delta\left(f-h f_{0}\right)\right) .
\end{aligned}
$$

If the width of the Lorentzian is much less than the fundamental frequency (i.e, $\tau^{-1} \ll f_{0}$ ), only one delta impulse in the comb makes a significant contribution to the convolution at each frequency, yielding

$$
\begin{aligned}
S(f) \propto & e^{-\pi|f| t_{w}}\left(1+e^{\frac{2 \pi i f\left(2 c-\frac{1}{2}\right)}{f_{0}}}\right) e^{-\frac{2 \pi i f c}{f_{0}}} \\
& \times \sum_{h=-\infty}^{\infty} \frac{\tau^{-1}}{\tau^{-2}+\left(2 \pi\left(f-h f_{0}\right)\right)^{2}}
\end{aligned}
$$

$$
\begin{aligned}
S(f) \propto & e^{-\pi|f| t_{w}} \cos \frac{\pi f\left(2 c-\frac{1}{2}\right)}{f_{0}} e^{-\frac{\pi i f}{2 f_{0}}} \\
& \times \sum_{h=-\infty}^{\infty} \frac{\tau^{-1}}{\tau^{-2}+\left(2 \pi\left(f-h f_{0}\right)\right)^{2}},
\end{aligned}
$$

and the amplitude for positive frequencies is

$$
\begin{aligned}
|S(f)| \propto & e^{-\pi f t_{w}}\left|\cos \frac{\pi f\left(2 c-\frac{1}{2}\right)}{f_{0}}\right| \\
& \times \sum_{h=1}^{\infty} \frac{\tau^{-1}}{\tau^{-2}+\left(2 \pi\left(f-h f_{0}\right)\right)^{2}} .
\end{aligned}
$$

\section{APPENDIX B: FOURIER TRANSFORM FOR A COHERENTLY DIFFUSING ION BUNCH}

$$
\begin{aligned}
s(t) & \propto e^{-|t| / \tau} \sum_{n=0}^{\infty} \frac{n \Delta T}{\frac{(n \Delta T)^{2}}{4}+t^{2}} \\
& \otimes\left(\delta\left(t-\frac{(n+c)}{f_{0}}\right)+\delta\left(t-\frac{\left(n+\frac{1}{2}-c\right)}{f_{0}}\right)\right) .
\end{aligned}
$$

With the ion bunch lengthening linearly in time, the Fourier transform for positive frequencies is

$$
\begin{aligned}
S(f) & \propto \frac{\tau^{-1}}{\tau^{-2}+(2 \pi f)^{2}} \\
& \otimes \sum_{n=0}^{\infty} e^{-\pi f n \Delta T}\left(e^{-\frac{2 \pi i f(n+c)}{f_{0}}}+e^{-\frac{2 \pi i f\left(n+\frac{1}{2}-c\right)}{f_{0}}}\right)
\end{aligned}
$$

Using the identity $\sum_{n=0}^{\infty} p^{n} e^{i n x}=\frac{1}{1-p e^{i x}}$,

$$
\begin{aligned}
S(f) & \propto \frac{\tau^{-1}}{\tau^{-2}+(2 \pi f)^{2}} \\
& \otimes \frac{e^{-2 \pi i f c / f_{0}}}{1-e^{-\pi f\left(f_{0} \Delta T+2 i\right) / f_{0}}}\left(1+e^{\frac{2 \pi i f\left(2 c-\frac{1}{2}\right)}{f_{0}}}\right) .
\end{aligned}
$$

For $f=h f_{0}+\delta f$, where $\delta f \ll f_{0}, c=0, \tau \rightarrow \infty$ and $h$ even

$$
\begin{gathered}
S(\delta f) \propto\left(1-e^{-\pi f \Delta T} e^{-2 \pi i \delta f / f_{0}}\right)^{-1} \\
|S(\delta f)| \propto\left(\left(1-e^{-\pi f \Delta T}\right)^{2}+e^{-\pi f \Delta T}\left(2 \pi \delta f / f_{0}\right)^{2}\right)^{-\frac{1}{2}} .
\end{gathered}
$$

\section{APPENDIX C: FREQUENCY DEPENDENCE OF THE FOURIER PHASE}

If we include additional time delay $\gamma / f_{0}$, and consider only imaginary multiplicative terms in Eq. (A3) we obtain

$$
S(f) \propto e^{-\frac{2 \pi i f(c+\gamma)}{f_{0}}}\left(1+e^{\frac{2 \pi i f\left(2 c-\frac{1}{2}\right)}{f_{0}}}\right),
$$




$$
\begin{gathered}
S(f) \propto\left(\cos \frac{2 \pi f(c+\gamma)}{f_{0}}-i \sin \frac{2 \pi f(c+\gamma)}{f_{0}}\right) \\
\left(1+\cos \frac{2 \pi f\left(2 c-\frac{1}{2}\right)}{f_{0}}+i \sin \frac{2 \pi f\left(2 c-\frac{1}{2}\right)}{f_{0}}\right), \\
S(f) \propto \cos \frac{2 \pi f\left(c-\frac{1}{4}\right)}{f_{0}} \\
\times\left(\cos \frac{2 \pi f\left(\gamma+\frac{1}{4}\right)}{f_{0}}-i \sin \frac{2 \pi f\left(\gamma+\frac{1}{4}\right)}{f_{0}}\right), \\
\phi(f)=-2 \pi \frac{f}{f_{0}}\left(\gamma+\frac{1}{4}\right) .
\end{gathered}
$$

\section{APPENDIX D: COMB-SAMPLING THEORETICAL RESOLUTION}

$$
\mathcal{S}(\delta f)=\frac{f_{0}}{M} \sum_{m=m_{1}}^{m_{2}}\left(\frac{k+p m-m \frac{|\delta f|}{f_{0}}}{(k+p m)^{2}}\right) .
$$

For no bunch diffusion $(p=0)$,

$$
\begin{gathered}
\mathcal{S}(\delta f)=\frac{f_{0}}{M k} \sum_{m=m_{1}}^{m_{2}}\left(1-m \frac{|\delta f|}{f_{0} k}\right)=\frac{f_{0}}{k}-\frac{|\delta f|}{M k^{2}} \sum_{m=m_{1}}^{m_{2}} \\
\mathcal{S}(\delta f)=\frac{f_{0}}{k}-\frac{|\delta f|}{2 M k^{2}}\left(m_{2}-m_{1}+1\right)\left(m_{2}+m_{1}\right), \\
\mathcal{S}(\delta f)=\frac{f_{0}}{k}\left(1-\frac{|\delta f|}{2 f_{0} k}\left(m_{2}+m_{1}\right)\right) .
\end{gathered}
$$

This expression [in Eq. (D4)] is only valid for

$$
\frac{m_{2}|\delta f|}{f_{0} k} \leq 1 \text {. }
$$

For coherent bunch diffusion $(k=0)$

$$
\mathcal{S}(\delta f)=\frac{f_{0}}{M p^{2}}\left(p-\frac{|\delta f|}{f_{0}}\right) \sum_{m=m_{1}}^{m_{2}} \frac{1}{m} .
$$

Using the following identity for an infinite harmonic series:

$$
\begin{gathered}
\lim _{m_{2} \rightarrow \infty} \sum_{m=1}^{m_{2}} \frac{1}{m}=\ln m_{2}+0.5772, \\
\mathcal{S}(\delta f)=\frac{f_{0}}{M p}\left(1-\frac{|\delta f|}{p f_{0}}\right) \ln \left(\frac{m_{2}}{m_{1}}\right), \quad \text { if } \quad m_{1}, m_{2} \gg 1,
\end{gathered}
$$

$$
\begin{aligned}
& \mathcal{S}(\delta f)=\frac{f_{0}}{M p}\left(1-\frac{|\delta f|}{p f_{0}}\right)\left(\ln m_{2}+0.5772\right), \\
& \text { if } m_{1}=1, m_{2} \gg 1 .
\end{aligned}
$$

\section{APPENDIX E: RESOLUTION OBTAINED WITH A LINEAR TIME WEIGHTING ON THE DATA}

For a signal decreasing linearly with a gradient of $\tau^{-1}$, $m_{1}=0$, and $p=0$,

$$
\begin{gathered}
\mathcal{S}(\delta f)=\frac{f_{0}}{M k} \sum_{m=0}^{m_{2}}\left(1-\frac{m}{k} \frac{|\delta f|}{f_{0}}\right)\left(1-\frac{m}{\tau f_{0}}\right), \\
\mathcal{S}(\delta f) \propto \sum_{m=0}^{m_{2}} 1-\frac{1}{\tau f_{0}} \sum_{m=0}^{m_{2}} m-\frac{|\delta f|}{k f_{0}}\left(\sum_{m=0}^{m_{2}} m-\frac{1}{\tau f_{0}} \sum_{m=0}^{m_{2}} m^{2}\right),
\end{gathered}
$$

$$
\begin{aligned}
& \mathcal{S}(\delta f) \propto m_{2}+1-\frac{1}{2 \tau f_{0}} m_{2}\left(m_{2}+1\right) \\
& -\frac{|\delta f|}{2 k f_{0}}\left(m_{2}\left(m_{2}+1\right)-\frac{1}{3 \tau f_{0}} m_{2}\left(m_{2}+1\right)\left(2 m_{2}+1\right)\right),
\end{aligned}
$$

$$
\mathcal{S}(\delta f) \propto 1-\frac{m_{2}}{2 \tau f_{0}}-\frac{|\delta f| m_{2}}{2 k f_{0}}\left(1-\frac{\left(2 m_{2}+1\right)}{3 \tau f_{0}}\right)
$$

$$
\mathcal{S}(\delta f) \propto 1-\frac{|\delta f| m_{2}\left(3 \tau f_{0}-2 m_{2}-1\right)}{3 k f_{0}\left(2 \tau f_{0}-m_{2}\right)} .
$$

Since $m_{2} \gg 1$, this gives a resolution of

$$
R=\frac{m_{2}\left(3 \tau f_{0}-2 m_{2}\right)}{6 k\left(2 \tau f_{0}-m_{2}\right)} .
$$

With a linearly increasing weighting ,

$$
\begin{gathered}
\mathcal{S}(\delta f) \propto \sum_{m=0}^{m_{2}} m-\frac{|\delta f|}{k f_{0}} \sum_{m=0}^{m_{2}} m^{2}, \\
\mathcal{S}(\delta f) \propto m_{2}\left(m_{2}+1\right)-\frac{|\delta f|}{3 k f_{0}} m_{2}\left(m_{2}+1\right)\left(2 m_{2}+1\right),
\end{gathered}
$$

$$
\mathcal{S}(\delta f) \propto 1-\frac{|\delta f|}{3 k f_{0}}\left(2 m_{2}+1\right) .
$$

Giving a resolution of $R=m_{2} / 3 k$ if $m_{2} \gg 1$.

${ }^{1}$ L. H. Andersen, O. Heber, and D. Zajfman, J. Phys. B 37, R57 (2004).

${ }^{2}$ K. H. Kingdon, Phys. Rev. 21, 408 (1923).

${ }^{3}$ S. P. Møller, Nucl. Instrum. Methods Phys. Res. A 394, 281 (1997).

${ }^{4}$ H. T. Schmidt, R. D. Thomas, D. Misra, M. Larsson, S. Rosén, P. Reinhed, H. Johansson, N. Haag, F. Seitz, H. Danared, P. Löfgren, A. Simonsson, K. G. Rensfelt, A. Källberg, L. Liljeby, H. Cederquist, J. Phys. Conf. Ser. 194, 142013 (2009).

${ }^{5}$ D. Zajfman, A. Wolf, D. Schwalm, D. A. Orlov, M. Grieser, R. von Hahn, C. P. Welsch, J. R. C. Lopez-Urrutia, C. D. Schröter, X. Urbain, and J. Ullrich, J. Phys.: Conf. Ser. 4, 296 (2005).

${ }^{6}$ J. Bernard, G. Montagne, R. Brédy, B. Terpend-Ordacière, A. Bourgey, M. Kerleroux, L. Chen, H. T. Schmidt, H. Cederquist, and S. Martin, Rev. Sci. Instrum. 79, 075109 (2008).

${ }^{7}$ A. Makarov, Anal. Chem. 72, 1156 (2000).

${ }^{8}$ W. H. Benner, Anal. Chem. 69, 4162 (1997).

${ }^{9}$ M. Dahan, R. Fisherman, O. Heber, M. Rappaport, N. Altstein, D. Zajfman, and W. J. van der Zande, Rev. Sci. Instrum. 69, 76 (1998). 
${ }^{10}$ H. T. Schmidt, H. Cederquist, J. Jensen, and A. Fardi, Nucl. Instrum. Methods Phys. Res. B 173, 523 (2001).

${ }^{11}$ J. D. Alexander, C. R. Calvert, R. B. King, O. Kelly, W. A. Bryan, G. R. A. J. Nemeth, W. R. Newell, C. A. Froud, I. C. E. Turcu, E. Springate, P. A. Orr, J. Pedregosa-Gutierrez, C. W. Walter, R. A. Williams, I. D. Williams, and J. B. Greenwood, J. Phys. B 42, 154027 (2009).

${ }^{12}$ H. B. Pedersen, D. Strasser, S. Ring, O. Heber, M. L. Rappaport, Y. Rudich, I. Sagi, and D. Zajfman, Phys. Rev. Lett. 87, 055001 (2001).

${ }^{13}$ O. Kelly, J. D. Alexander, C. R. Calvert, R. B. King, L. Graham, I. D. Williams, J. B. Greenwood, W. R. Newell, W. A. Bryan, G. R. A. J. Nemeth, E. Springate, C. A. Froud, and I. C. E. Turcu, Central Laser Facility Annual Report: Femtosecond Pulse Physics, 2008/2009.

${ }^{14}$ Image adapted from simion ${ }^{\circledR}$ 8.0, Ion Optics Simulation Software, Scientific Instrument Services, Inc.

${ }^{15}$ J. D. Alexander, L. Graham, C. R. Calvert, O. Kelly, R. B. King, I. D. Williams, and J. B. Greenwood, Meas. Sci. Technol. 21, 045802 (2010).

${ }^{16}$ I. C. E. Turcu, E. Springate, C. A. Froud, C. M. Cacho, J. L. Collier, W. A. Bryan, G. R. A. J. Nemeth, J. P. Marangos, J. W. G. Tisch, R. Torres, T. Siegel, L. Brugnera, J. G. Underwood, I. Procino, W. R. Newell, C. Altucci, R. Velotta, R. B. King, J. D. Alexander, C. R. Calvert, O. Kelly,
J. B. Greenwood, I. D. Williams, A. Cavalleri, J. C. Petersen, N. Dean, S. S. Dhesi, L. Poletto, P. Villoresi, F. Frassetto, S. Bonora, and M. D. Roper, Proc. SPIE 7469, 746902 (2010).

${ }^{17}$ H. B. Pedersen, D. Strasser, B. Amarant, O. Heber, M. L. Rappaport, and D. Zajfman, Phys. Rev. A 65, 042704 (2002).

${ }^{18}$ A. Piechaczek, V. Shchepunov, H. K. Carter, J. C. Batchelder, E. F. Zganjar, S. N. Liddick, H. Wollnik, Y. Hu, and B. O. Griffith, Nucl. Instrum. Methods Phys. Res. B 266, 4510 (2008).

${ }^{19}$ S. Ring, H. B. Pedersen, O. Heber, M. L. Rappaport, P. D. Witte, K. G. Bhushan, N. Altstein, Y. Rudich, I. Sagi, and D. Zajfman, Anal. Chem. 72, 4041 (2000).

${ }^{20}$ K. G. Bhushan, S. C. Gadkari, J. V. Yakhmi, and V. C. Sahni, Rev. Sci. Instrum. 78, 083302 (2007).

${ }^{21}$ J. U. Andersen, P. Hvelplund, S. B. Nielsen, S. Tomita, H. Wahlgreen, S. P. Møller, U. V. Pedersen, J. S. Forster, and T. J. D. Jørgensen, Rev. Sci. Instrum. 73, 1284 (2002).

${ }^{22}$ C. L. Hendrickson, S. C. Beu, G. T. Blakney, and A. G. Marshall, Int. J. Mass Spectrom. 283, 100 (2009).

${ }^{23}$ P. A. Limbach, P. B. Grosshans, and A. G. Marshall, Int. J. Mass Spectrom. Ion Process. 123, 41 (1993).

${ }^{24}$ E. N. Nikolaev, V. S. Rakov, and J. H. Futrell, Int. J. Mass Spectrom. Ion Process. 157-158, 215 (1996). 